\title{
Effects of Zhuyu Annao on Hippocampal Neuronal Apoptosis and Cognitive Disorder in a Rat Model of Ischemic Brain Injury
}

\section{Type}

Research paper

\section{Keywords}

hippocampus, Neuronal apoptosis, ischemic brain injury, cognitive disorder, Zhuyu Annao prescription

\begin{abstract}
Introduction

We aimed to determine the effects of Zhuyu Annao (ZYAN) prescription on hippocampal neuronal apoptosis and cognitive disorder (CD) in ischemic brain injury (IBI).

\section{Material and methods}

The improved Rice-Vannucci method was used to prepare a rat model of IBI. Rats in the ZYAN prescription group were intragastrically administered $12.50 \mathrm{~g} / \mathrm{kg}$ of ZYAN prescription $4 \mathrm{~h}$ after modeling; rats in the mock surgical group (MSG) and model group (MG) were administered an intraperitoneal injection of the same volume of normal saline twice daily.
\end{abstract}

\section{Results}

Compared with MG, the area of cerebral infarction, pathological changes, neuron apoptosis index, and neurodeficit score were significantly reduced in the ZYAN prescription group $(P<0.05)$. Morris water maze test showed that in the ZYAN prescription group, incubation period was significantly shortened and the number of platform crossings was significantly increased $(P<0.05)$. Furthermore, the rats in the ZYAN prescription group showed significantly reduced protein and mRNA expressions of cleaved caspase-3, Bax, p-53, and p38 mitogen-activated protein kinase (MAPK) in the hippocampus, whereas the protein and mRNA expressions of Bcl-2 were significantly increased $(\mathrm{P}<$ 0.05).

\section{Conclusions}

ZYAN prescription reduced the area of cerebral infarction, attenuated neuronal apoptosis, and alleviated CD in the rats with IBI. The beneficial effect of ZYAN prescription in IBI may be mediated via the inhibition of the p38 MAPK signaling pathway and caspase-3 cascade reaction. 
Effects of Zhuyu Annao on Hippocampal Neuronal Apoptosis and Cognitive

\section{Disorder in a Rat Model of Ischemic Brain Injury}

Wei Chen ${ }^{1,2,}$, Lingfei Jiang ${ }^{1,2, \S}$, Yueqiang $\mathrm{Hu}^{1,2}$, Nong Tang ${ }^{1,2}$, Ni Liang ${ }^{1}$, Xing-Feng

$\mathrm{Li}^{3}$, Ye-Wen Chen ${ }^{3}$, Hongling $\mathrm{Qin}^{1}$, Lin $\mathrm{Wu}^{1,2,4, *}$

1 Department of Neurology, The First Affiliated Hospital of Guangxi University of

Chinese Medicine, Nanning, Guangxi, China

2 Guangxi Key Laboratory of Chinese Medicine Foundation Research, Guangxi

University of Chinese Medicine, Nanning, Guangxi, China

3 Graduate College of Guangxi University of traditional Chinese Medicine, Nanning,

Guangxi, China

4 Scientific Laboratorial Centre Guangxi University of Chinese Medicine, Nanning, Guangxi, China

${ }^{\S}$ Wei Chen and Lingfei Jiang are co-first authors.

\section{*Corresponding author}

\section{Lin $\mathrm{Wu}$}

Department of Neurology, The First Affiliated Hospital of Guangxi University of

Chinese Medicine, No. 89-9, Gedong Road, Nanning, 530023 Guangxi, China

Tel: 86-0771-5848502

Email: y7qf8y@163.com

\section{Authors Email}


Wei Chen

Lingfei Jiang

Yueqiang $\mathrm{Hu}$

Nong Tang

Ni Liang

Xing-Feng Li

Ye-Wen Chen

Hongling Qin

Lin $\mathrm{Wu}$
Email: chenwei8126@163.com

Email:297505147@qq.com

Email: Shitousay1111@tom.com

Email:ntang@gxtcmu.edu.cn

Email: nizilinjia@126.com

Email: Lixingfeng5200@126.com

Email: chen18070932468@163.com

Email:286269060@qq.com

Email: y7qf8y@163.com

Running title: Effects of Zhuyu Annao

\section{Abstract}


Introduction: We aimed to determine the effects of Zhuyu Annao (ZYAN) prescription on hippocampal neuronal apoptosis and cognitive disorder (CD) in ischemic brain injury (IBI). Methods: The improved Rice-Vannucci method was used to prepare a rat model of IBI. Rats in the ZYAN prescription group were intragastrically administered $12.50 \mathrm{~g} / \mathrm{kg}$ of ZYAN prescription $4 \mathrm{~h}$ after modeling; rats in the mock surgical group (MSG) and model group (MG) were administered an intraperitoneal injection of the same volume of normal saline twice daily. Results: Compared with MG, the area of cerebral infarction, pathological changes, neuron apoptosis index, and neurodeficit score were significantly reduced in the ZYAN prescription group $(P<0.05)$. Morris water maze test showed that in the ZYAN prescription group, incubation period was significantly shortened and the number of platform crossings was significantly increased $(P<0.05)$. Furthermore, the rats in the ZYAN prescription group showed significantly reduced protein and mRNA expressions of cleaved caspase-3, Bax, p-53, and p38 mitogen-activated protein kinase (MAPK) in the hippocampus, whereas the protein and mRNA expressions of Bcl-2 were significantly increased $(P<0.05)$. Conclusions: ZYAN prescription reduced the area of cerebral infarction, attenuated neuronal apoptosis, and alleviated CD in the rats with IBI. The beneficial effect of ZYAN prescription in IBI may be mediated via the inhibition of the p38 MAPK signaling pathway and caspase-3 cascade reaction.

Keywords: Zhuyu Annao prescription; ischemic brain injury; hippocampus; neuronal apoptosis; cognitive disorder 


\section{Introduction}

Ischemic stroke (IS) is one of the main causes of disability and death in adults.

According to estimates, a case of symptomatic stroke is observed every $2 \mathrm{~s}$; therefore, it is considered as one of the major public health issues [1,2]. Currently, common treatment methods for IS include thrombolysis, reduction of intracranial pressure, use of neuroprotective agents, and removal of hematoma. However, treatment efficacy is typically poor because of a short therapeutic time window and adverse effects of drugs; thus, approximately $70 \%-80 \%$ stroke survivors suffer from cognitive disorder (CD), which seriously affects their prognosis and quality of life [3-5]. Therefore, elucidation of the pathogenic mechanisms underlying post-IS neuronal damage and $\mathrm{CD}$ and development of more effective therapies are key research imperatives.

A correlation has been demonstrated between ischemic brain injury (IBI) and mitochondrial dysfunction, cell apoptosis, oxygen radical activation, and inflammatory reaction [6]. Necrosis and apoptosis are two forms of neuronal death, with apoptosis being the main form of neuronal death in IBI. Other studies have revealed that the hyperphosphorylation of the hippocampal Tau protein is closely associated with CD [7]. Zhuyu Annao (ZYAN) prescription is a traditional Chinese medicine (TCM)-formula granule manufactured by Jiangyin Tian Jiang Pharmaceutical Co. Ltd. It is based on the ZYAN pill developed by chief physician Wu Zihui (a distinguished veteran TCM practitioner in Guangxi). ZYAN prescription has been widely used in the treatment of craniocerebral injury and $\mathrm{CH}$ encephaledema. 
Increasing evidence has shown that the ZYAN prescription may alleviate IBI through the inhibition of cell apoptosis and anti-inflammatory action [8]. In a preliminary clinical study, neurological function defect score, cerebral hematoma absorption rate, and cerebral edema were significantly improved in the acute stage of hemorrhagic stroke; the total effective rate and basic cure rate were $92.50 \%$ and $47.50 \%$, respectively [9]. The p38 mitogen-activated protein kinase (MAPK) signaling pathway and caspase- 3 cascade reaction are the most common mechanisms involved in neuronal apoptosis. Based on these findings, we hypothesized that the ZYAN prescription plays a role in improving neuronal apoptosis in IBI through the activation of the p38 MAPK signaling pathway and caspase-3 cascade reaction. In this study, we sought to explore new targets for the ZYAN prescription and elucidate its mechanism of action. In particular, we investigated the effects of the ZYAN prescription on hippocampal nerve cell apoptosis and CD in rats with IBI; additionally, we explored its potential neuroprotective effect to provide evidence for clinical practice.

\section{Material and Methods}

\section{Material}

A total of 72 clean-grade male Sprague-Dawley rats (age: 18 months; weight:

270-300 g) were purchased from Laboratory Animal Centre, Chongqing Medical

University. The rats were given ad libitum access to food and water and were housed in a controlled environment (temperature: $21^{\circ} \mathrm{C}-23^{\circ} \mathrm{C}$; relative humidity: $40 \%-60 \%$ ). 
The rats were randomly assigned to a mock surgical group (MSG; $n=24$ ), model group (MG; $n=24)$, or ZYAN prescription group $(n=24)$ using the random number table method. All procedures were approved by the Animal Care and Use Committee of The First Affiliated Hospital of Guangxi University of Chinese Medicine.

\section{Reagents used}

The ZYAN prescription was developed into a granule formula (Hirudo; woodlouse; earthworm, $10 \mathrm{~g}$; peach seed, $10 \mathrm{~g}$; Campsis grandiflora, $10 \mathrm{~g}$; Herba lycopi, $10 \mathrm{~g}$; wine-treated rhubarb, 10 g; Rhizoma chuanxiong, 10 g; Polyporus umbellatus, $10 \mathrm{~g}$; and Rhizoma alismatis, $10 \mathrm{~g}$ ) by Jiangyin Tian Jiang Pharmaceutical Co. Ltd. Cytochrome C, Taul, and PT231 antibodies were produced by Chemicon. Morris water maze system was purchased from Shanghai Jiliang Software Technology Co. Ltd.; triphenyltetrazolium chloride (TTC) from Shanghai Hualan Chemical Technology Co. Ltd.; Nissl staining solution from Beijing Solarbio Science \& Technology Co. Ltd.; TUNEL assay kit from Roche; cell counting kit-8 from Dojindo; SDS-PAGE kit from Wuhan Servicebio Technology Co. Ltd.; BCA protein assay kit from Bio-rad; phenylmethylsulfonyl fluoride, phosphorylase inhibitor, PIRA lysate, developer, and fixer kit for black and white film and paper and ECL from Wuhan Servicebio Technologies Co. Ltd.; polyvinylidene fluoride membrane from Millipore; and $\beta$-actin antibody, cleaved caspase-3 (activated), monoclonal antibody (rabbit anti-rat), Bcl-2 monoclonal antibody (rabbit anti-rat), Bcl 2-associated X protein monoclonal antibody (rabbit anti-rat), p53 (rabbit anti-rat), p-p38 monoclonal antibody (rabbit anti-rat), and MAPK monoclonal antibody (rabbit anti-rat) from 
Sigma.

\section{Methods}

\section{Establishment of animal models and administration methods}

The improved Rice-Vannucci method [10] was used to prepare the rat model of IBI. Except for the rats in MSG, those in MG and the ZYAN prescription group were anesthetized via inhalation of diethyl ether. Post anesthesia, the rats were placed in supine position, and their neck skin was exposed and incised layer by layer to separate the tissues. Then, in the rats of all the groups, except those of MSG, the common carotid arteries of both sides were exposed, ligated, and sutured. After successful modeling, the rats in the ZYAN prescription group were intragastrically administered $12.50 \mathrm{~g} / \mathrm{kg}$ of the ZYAN prescription twice a day through the mouth using a 20 -gauge gavage needle. The rats in MSG and MG were intraperitoneally administered the same concentration of normal saline. All the groups were administered the indicated drugs for 7 consecutive days. The grouping and dosage administered to the rats are shown in Table 1.

\section{Neurologic deficit and behavioral test}

Eight rats were selected from each group, and the Morris water maze (MWM) method was used to assess behavioral changes. The MWM apparatus consists of a circular pool measuring $120 \times 60 \mathrm{~cm}$, with a cylindrical $10 \times 40$-cm PMMA platform above it. The room and water temperatures were maintained at $24^{\circ} \mathrm{C}-26^{\circ} \mathrm{C}$. MWM training was conducted 5 days before the modeling and four times daily 1, 2, 3, 4, 5, 6, and 7 
days after the modeling. During each training, the rats were placed in the water with their head toward the pool wall, at a half radian of the quadrant farthest from the platform; the route taken by the rats in the pool before they found the platform in the water was observed, and this period was termed as incubation period (IP). The longest swimming time was $<90 \mathrm{~s}$. The platform was removed after the rats found it. The rats were subsequently placed at the midpoint of the quadrant opposite to the platform denoted as the crossing platform to record the number of platform crossings performed by the rats within $90 \mathrm{~s}$. In addition, the Garcia reporting method was used for scoring the nerve function 7 days after the administration of drugs, and the following aspects were evaluated: autonomic activity, symmetrical motion, symmetrical abduction of forelimbs, climbing, response to touching of body with a blunt rod, and response to touching of whiskers with a blunt rod. The scores ranged from 3 to 18 (18 representing normal function; the higher the scores, the lesser the nerve function defect).

\section{Measurement of the area of cerebral infarction using TTC}

After administration of the drugs for 7 days, eight rats in each group were anesthetized using an intraperitoneal injection of $10 \%$ chloral hydrate $(3.50 \mathrm{~mL} / \mathrm{kg})$. Subsequently, the rats were decapitated, their brains were collected, and 2-mm-thick coronal brain slices were prepared. The slices were immersed in 2\% TTC staining solution and subsequently incubated in the dark at $37^{\circ} \mathrm{C}$ for 20 min. PBS buffer solution was used to wash off the TTC solution, after which the slices were placed in $4 \%$ paraformaldehyde and fixed in the dark at $4{ }^{\circ} \mathrm{C}$. Images were captured after $24 \mathrm{~h}$. 
Image $\mathbf{J}$ software was used to analyze the area of cerebral infarction; the percentage of the area of cerebral infarction was calculated using the following formula:

area of cerebral infarction $\%=($ left cerebral hemisphere size - size of noninfarcted right cerebral hemisphere) $/ 2 \times$ size of left cerebral hemisphere $\times 100$

\section{Preparation of tissue slices and Nissl staining}

After administration of the drugs for 7 days, eight rats selected from each group were anesthetized. The rats were decapitated, and their brains were collected. The olfactory bulb, cerebella, and lower brain stem were removed. The brain tissues were immersed in $4 \%$ paraformaldehyde, fixed for $60 \mathrm{~min}$, and soaked in $30 \%$ sucrose solution.

Subsequently, 5- $\mu \mathrm{m}$-thick slices were prepared and used for Nissl staining, immunohistochemical staining, and cell apoptosis detection. The Nissl staining revealed the pathological changes in the tissues, which were examined under a light microscope.

\section{Detection of hippocampal neuron apoptosis by terminal deoxynucleotidyl transferase dUTP nick end labeling (TUNEL) staining method}

As per the TUNEL assay kit's instructions, the tissues were stained using a DAPI working solution for $10 \mathrm{~min}$ at $37^{\circ} \mathrm{C}$ and washed thrice with PBS buffer solution for 5 min. Subsequently, the slices were sealed and fixed. A fluorescence microscope was used to detect hippocampal neuron apoptosis; the number of TUNEL-positive (apoptotic) cells was observed under a high-power field $(\times 400)$. A total of 200 cells were counted, and the number of normal cells differing in their nuclei from those of 
the apoptotic cells was recorded.

Apoptosis index $(\mathrm{AI})=$ number of TUNEL-positive cells $/$ total number of cells $\times$ $100 \%$. Five fields were randomly selected from each slice, and the mean AI value was used in the analysis.

\section{Western blot}

The brain tissues were excised and homogenized on ice. A protein extraction buffer was used for total protein extraction. Then, the BCA assay method was used to determine the protein concentration and calculate the sample loading quantity. Separation and spacer gels were prepared, followed by SDS-PAGE electrophoresis, membrane transfer, sealing, and incubation with primary antibodies (Taul, referring to goat anti-rat, and PT231, referring to goat anti-rabbit) and secondary antibodies (goat anti-rat IgG with fluorescence labeling, 800 pathway). Finally, grayscale values were detected using Odyssey ${ }^{\circledR}$ Infrared Imaging program (LI-COR Biosciences).

\section{Fluorescence quantitative polymerase chain reaction (PCR)}

The brain tissues were removed, frozen in liquid nitrogen, and ground into powder. Total RNA was extracted with TRIZOL reagent for quantitative analysis. After determining that the A260/A280 was 1.8-2.0, $1 \mathrm{ng}$ of the RNA sample, $1 \mu \mathrm{L}$ oligo (dT), RNase-free $\mathrm{dH}_{2} \mathrm{O}, 0.5 \mu \mathrm{L}$ dNTP mixture, $2 \mu \mathrm{L} 5 \times$ M-MLV buffer, $0.25 \mu \mathrm{L}$ RTase M-MLV, and $0.25 \mu \mathrm{L}$ RNase inhibitor were used to prepare the sample, which was then incubated at $42^{\circ} \mathrm{C}$ for $1 \mathrm{~h}$, followed by $70^{\circ} \mathrm{C}$ for $15 \mathrm{~min}$, ice for $5 \mathrm{~min}$. Subsequently, PCR detection was performed. The PCR reaction mixture comprised 50 
ng cDNA, $0.5 \mu \mathrm{L}$ upstream and downstream primers, $25 \mu \mathrm{L}$ FastStart Universal SYBR Green Master (ROX), and RNase-free $\mathrm{dH}_{2} \mathrm{O}$, made up to $50 \mu \mathrm{L}$ of sample volume. The reaction conditions were as follows: predenaturation at $95^{\circ} \mathrm{C}$ for $10 \mathrm{~min}$, denaturation at $95^{\circ} \mathrm{C}$ for $10 \mathrm{~s}$, annealing at $60^{\circ} \mathrm{C}$ for $20 \mathrm{~s}$, and extension at $72^{\circ} \mathrm{C}$ for 15 s, performed for a total of 40 cycles. $2^{-\Delta \Delta \mathrm{Ct}}$ was used to calculate the mRNA levels of cleaved caspase-3, Bax, p53, p38 MAPK, and Bcl-2. The primer sequences used were as follows:

cleaved caspase-3: Forward: 5'-GCAGCAGCCTCAAATTGTTGAC-3', Reverse 5'-TGCTCCGGCTCAAACCATC-3', 205 bp; Bax: Forward 5'-GGATGCGTCCACCAAGAA-3', Reverse 5'-TCCCGGAGGAAGTCCATT-3', 148 bp; Bcl-2: Forward 5'-CCTTTGTGTAACTGTACGGCC-3', Reverse 5'-CTTTGGCAGTAAATAGCTGATTCGAC-3', 270 bp; p53: Forward 5'-TATGGCTTCCACCTGGGCTT-3', Reverse 5'-TCTTCCAGATACTCGGGATACAA-3', 306 bp; p38 MAPK: Forward 5'-TGCCCGAACGTACCAGAAC-3', Reverse 5'-CCTTTTGGCGTGAATGATGGA-3', 142 bp; $\beta$-actin; Forward 5'-CTCCATCCTGGCCTCGCTG-3', Reverse 5'-GCTGTCACCTTCACCGTTCC-3', $268 \mathrm{bp}$. The primers were synthesized by Sangon Biotech (Shanghai) Co. Ltd.

\section{Statistical analysis}

All data analyses were performed using SPSS 25.0 statistical software. Continuous variables are presented as mean \pm standard deviation (mean $\pm \mathrm{SD}$ ), and between-group differences were assessed using one-way ANOVA. For pair-wise 
comparisons, the LSD method was used in case of homogenous variance, whereas the Dennett T3 method was used in case of heterogeneous variance. $P<0.05$ was considered statistically significant.

\section{Results}

\section{ZYAN prescription improves neurodeficit score (NDS) and spatial learning and memory ability (LMA)}

As shown in Figure 1A, compared with the rats in MSG, NDS was significantly reduced in MG; however, NDS was significantly increased in the ZYAN prescription group compared with that in MG $(P<0.05)$, implying that the ZYAN prescription alleviates nerve defect in rats with IBI.

In the MWM test, the IP of MG was significantly longer than that of MSG on days 2 and 3; on day 7, the IP was significantly shorter in the ZYAN prescription group than that in MG $(P<0.05$; Figure 1B-D). Moreover, compared with MSG, the number of platform crossings were significantly increased in MG but were significantly reduced in the ZYAN prescription group compared with that in MG $(P<0.05)$, implying that the ZYAN prescription improves spatial LMA in rats with IBI and promotes the recovery of nerve injury.

\section{ZYAN prescription reduces the area of cerebral infarction}

Approximately $48 \mathrm{~h}$ after the modeling, the rats were decapitated and their brains collected. The brains appeared abnormal (Figure 2A); in MG and the ZYAN 
prescription group, both sides of the brain were asymmetrical. In addition, there was obvious edema and local whiteness in the right cerebral hemisphere; however, in MSG, both sides of the brain were symmetrical, with no whiteness or edema. TTC staining revealed obvious infarction in the brain tissues of MG and the ZYAN prescription group but not in those of MSG. As shown in Figure 2B-C, compared with MSG, the brain water content (BWC) and area of cerebral infarction were significantly higher in MG and the ZYAN prescription group $(P<0.05)$; however, the BWC and area of cerebral infarction were significantly lower in the ZYAN prescription group than in MG $(P<0.05)$, implying that the ZYAN prescription significantly reduces the cerebral edema volume and the area of cerebral infarction in rats with IBI.

\section{ZYAN prescription alleviates hippocampal nerve cell damage}

According to the Nissl staining results obtained $48 \mathrm{~h}$ after the modeling, in MSG, the nerve cells in the hippocampus CA1 and cortical areas were arranged in neat rows; in addition, pyramid-shaped cells with a clear structure were observed, and an ianthinus Nissl body was observed in the cytosol. However, in MG and the ZYAN prescription group, the nerve cells in the hippocampus CA1 and cortical areas showed ischemic changes; there appeared to be cellular edema with a loose and irregular tissue structure. Moreover, the Nissl body was not observed in some nerve cells (Figure 3A-B). Based on the statistical analysis, the number of Nissl-positive cells in the hippocampus CA1 and cortical areas were reduced in the MG and the ZYAN prescription group compared with that in MSG; however, the number of 
Nissl-positive cells were significantly increased in the ZYAN prescription group compared with that in MG $(P<0.05$, Figure 3C-D), implying that the ZYAN prescription reduces ischemic injury of hippocampal nerve cells and restores their normal structure in rats with IBI.

\section{ZYAN prescription inhibits hippocampal neuronal apoptosis}

In Figure 4, the apoptotic cells show brown nuclear staining or deposits of brown particles. Compared with MSG, the neuron count and AI in the hippocampus CA1 were significantly higher in MG and the ZYAN prescription group $(P<0.05)$; however, the neuron count and AI in the ZYAN prescription group were significantly lower than that in MG $(P<0.05)$, implying that the ZYAN prescription inhibits hippocampal neuronal apoptosis and promotes the recovery of neuron injury.

\section{ZYAN prescription reduces the expression of apoptosis-related proteins}

As shown in Figure 5, compared with MSG, the protein expression levels of cleaved caspase-3, Bax, p53, and p38 MAPK in the hippocampus were significantly increased in MG; however, the protein expression level of Bcl-2 in MG was significantly reduced $(P<0.05)$. Compared with $\mathrm{MG}$, the protein expression levels of cleaved caspase-3, Bax, p53, and p38 MAPK in the hippocampus were significantly reduced and the protein expression level of Bcl-2 was significantly increased in the ZYAN prescription group $(P<0.05)$, implying that the ZYAN prescription significantly reduces the levels of caspase-3, Bax, p53, and p38 MAPK proteins in the hippocampal tissues and increases the levels of Bcl-2, thereby inhibiting neuronal 
apoptosis.

\section{ZYAN prescription inhibits the expression of apoptosis-related genes}

As per the fluorescent quantitative (FQ)-PCR results, the mRNA expression levels of cleaved caspase-3, Bax, p53, and p38 MAPK in the hippocampus were significantly increased and that of Bcl-2 was significantly reduced in MG $(P<0.05)$. Compared with MG, the mRNA expression levels of cleaved caspase-3, Bax, p53, and p38 MAPK in the hippocampus were significantly reduced and that of Bcl-2 was significantly increased in the ZYAN prescription group $(P<0.05)$, implying that the ZYAN prescription inhibits the expression of apoptosis-related genes in the hippocampal neurons. These results were consistent with the Western blot results (Figure 6).

\section{Discussion}

IS can cause damage to the neurons and white matter, which is the main reason for death and neurologic deficit in patients with IS [11]. Currently, a large number of basic and clinical studies on IS are ongoing [12]. Although the pathogenesis of IS has been understood to a certain extent, the pathophysiological process of IS involves complex cascade reactions in time and space; thus, there is a paucity of effective treatment modalities for IS. In this study, we investigated the effects of the ZYAN prescription on cognitive impairment and apoptosis of the hippocampus nerve cells in IBI. The results showed that the ZYAN prescription reduced neuronal apoptosis and 
alleviated cognitive impairment in the rats with IBI.

Chen W et al. [13] have indicated that Toll-like receptor 4 (TLR4) mediates the inflammatory injury caused by $\mathrm{CH}$. In addition, the ZYAN prescription exhibited neuroprotective effects by reducing TLR4 expression to alleviate encephaledema and neuronal apoptosis. In this study, we established a rat model of cerebral ischemia (CI) and assessed the effects of the ZYAN prescription on the model. Compared with MSG, MG exhibited deterioration in $\mathrm{CD}$, with a significant increase in the area of cerebral infarction, hippocampal nerve cell damage index, and hippocampal nerve cell AI; however, the ZYAN prescription significantly alleviated CD, with a decrease in the area of cerebral infarction, hippocampal nerve cell damage index, and hippocampal nerve cell AI. These findings indicate a neuroprotective effect of the ZYAN prescription in IBI, which is consistent with the abovementioned reports. However, the mechanism by which the ZYAN prescription alleviates neuronal apoptosis and improves CD remains unclear.

CI-mediated cell apoptosis is broadly categorized as occurring via exogenous or endogenous pathways. The exogenous pathway of cell apoptosis is mediated via a cascade reaction involving cysteinyl aspartate specific proteinase (caspase)-8, caused by the activation of death receptors on the cell surface. The endogenous pathway, also known as mitochondria-mediated cell apoptosis, is the main pathway of neuronal apoptosis in CI; it mainly refers to the cell apoptosis caused by the activation of caspase-3 after mitochondrial stimulation $[14,15]$. Cell apoptosis is accomplished mainly via caspase activation. Caspase-3 can permeate the cell nucleus by 
hydrolyzing the arrestin of deoxyribonucleases, leading to activation of key factors downstream of the caspase cascade reactions, thereby resulting in cell apoptosis. Simultaneously, caspase- 3 can dissociate from Bcl-2, causing a Bax/Bcl-2 imbalance, which promotes neuronal apoptosis [16-18]. Furthermore, neuronal apoptosis is also regulated by the p38 MAPK signaling pathway; activation of this pathway can directly activate Bax and transport it to the outer mitochondrial membrane, thereby mediating the entry of cytochrome $\mathrm{C}$ into the cytoplasm, activating the caspase- 3 cascade reaction $[19,20]$. Liu Y et al. [21] have found that changes in mitochondrial function are closely related to neuronal apoptosis. In addition, the more severe the neuronal apoptosis, the more obvious the increase in the expression of the mitochondrial apoptosis-related genes caspase-3, Bax, and Bcl-2 [22]. Liang [23] has found that the Annao pill significantly reduces microglial cell activation and the expression of NF- $\mathrm{KBp} 65$ and caspase-3 after ICH; in addition, it increases the expression of a brain-derived neurotrophic factor and synaptophysin. These changes exert a protective effect on hemorrhagic brain tissue. He et al. [24] have shown that the Naoyian granule can inhibit DNA fragmentation in nerve cells and reduce neuronal apoptosis. These studies suggest that TCM preparations can significantly reduce neuronal apoptosis and play a protective role against brain injury. In our study, compared with MG, the ZYAN prescription group showed a significantly lower protein expression of cleaved caspase-3, Bax, p-p38, and MAPK in the hippocampus, and a significantly higher protein expression of $\mathrm{Bcl}-2$; these findings were consistent with those of FQ-PCR. Therefore, we hypothesized that the ZYAN prescription 
inhibits neuronal apoptosis by inhibiting the p38 MAPK signaling pathway and caspase-3 cascade reaction. The ZYAN prescription contains Hirudo, woodlouse, $C$. grandiflora, peach seed, earthworm, $R$. chuanxiong, wine-treated rhubarb, and other drugs. In previous studies, the chemicals contained in the abovementioned components, such as hirudin [25], ligustrazine [26], and chrysophanol [27], have been found to improve brain injury and brain edema caused by CI. The results of this study are consistent with those of previous studies.

However, some limitations of our study should be considered while interpreting our results. In this study, a rat model was developed, and TTC staining was used as the classic method to detect the areas of cerebral infarction in the rats. The coordinates where the infarcts were the most obvious in each group were selected. Due to individual differences in the rats and an error in experimental operation, it was difficult to ensure the same infarcts in the same coordinates of the brain. Therefore, the most obvious ischemic lesions of the rat brain were selected for comparison. Due to the effect of photography, dyeing, and photo pixels, the image may only show a local whitish discoloration and no obvious infarction lesions. Therefore, the results of this experiment may not be very convincing. Moreover, the relationship between the effect of the individual active components of the ZYAN prescription on IBI and that of the combined prescription has not been studied; a confirmatory study will be performed in the future.

\section{Conclusions}

In conclusion, the ZYAN prescription was found to reduce neuronal apoptosis and the 
area of cerebral infarction and improve $\mathrm{CD}$ in the rats with IBI. The underlying mechanism of action of the ZYAN prescription may be related to the inhibition of neuronal apoptosis by ZYAN via the inhibition of the p38 MAPK signaling pathway and caspase- 3 cascade reaction.

\section{List of abbreviations}

ZYAN: Zhuyu Annao

CD: cognitive disorder

IBI: ischemic brain injury

MSG: mock surgical group

MG: model group

MWM: Morris water maze

MAPK: mitogen-activated protein kinase

IS: ischemic stroke

TCM: traditional Chinese medicine

$\mathrm{CH}$ : cerebral hemorrhage

TTC: triphenyltetrazolium chloride

CCK-8: cell counting kit-8

PMSF: phenylmethylsulfonyl fluoride

PVDF: polyvinylidene fluoride

NS: normal saline

IP: incubation period 
AI: apoptosis index

NDS: neurodeficit score

LMA: learning and memory ability

TLR4: Toll-like receptor 4

CI: cerebral ischemia

Acknowledgment: This study was funded by the National Natural Science

Foundation (grant number: 81760847); The Key Research and Development Program of Science and Technology Plan Project of Guangxi (grant number:

GuikeAB16380324); the Project of Guangxi Key Laboratory of Chinese Medicine Foundation Research (grant number: K558); the Youth Innovation Research Team of Guangxi University of Traditional Chinese Medicine (grant number: 2016QT004); and the High-level Talent Team Cultivation Project of Qihuang Project of Guangxi University of Traditional Chinese Medicine (grant number: 2018003).

Conflict of Interest: The authors declare that they have no conflict of interest.

\section{References}

1. Khanevski AN, Bjerkreim AT, Novotny V, et al. Recurrent ischemic stroke: Incidence, predictors, and impact on mortality. Acta Neurol Scand 2019;140: 3-8.

2. Devyatov AA, Fedorova TN, Stvolinskii SL, Belousova MA, Medvedev OS, Tutelyan VA. Assessment of Oxidative Status of the Brain and Blood Plasma in 
Rats with Modeled Focal Cerebral Ischemia/Reperfusion Injury. Bull Exp Biol Med 2017;163: 195-8.

3. Zhang X, Shen X, Dong J, et al. Inhibition of Reactive Astrocytes with Fluorocitrate Ameliorates Learning and Memory Impairment Through Upregulating CRTC1 and Synaptophysin in Ischemic Stroke Rats. Cell Mol Neurobiol 2019;39: 1151-63.

4. Liang Y, Chen YK, Liu YL, et al. Cerebral Small Vessel Disease Burden Is Associated With Accelerated Poststroke Cognitive Decline: A 1-Year Follow-Up Study. J Geriatr Psychiatry Neurol 2019;32: 336-43.

5. Escobar I, Xu J, Jackson CW, Perez-Pinzon MA. Altered Neural Networks in the Papez Circuit: Implications for Cognitive Dysfunction after Cerebral Ischemia. J Alzheimers Dis 2019;67: 425-46.

6. Sekerdag E, Solaroglu I, Gursoy-Ozdemir Y. Cell Death Mechanisms in Stroke and Novel Molecular and Cellular Treatment Options. Curr Neuropharmacol 2018;16: 1396-415.

7. Shahpasand K, Sepehri Shamloo A, Nabavi SM, Ping Lu K, Zhen Zhou X. "Tau immunotherapy: Hopes and hindrances". Hum Vaccin Immunother 2018;14: 277-84.

8. Feng D, Xia Z, Zhou J, et al. Metabolomics reveals the effect of Xuefu Zhuyu Decoction on plasma metabolism in rats with acute traumatic brain injury. Oncotarget 2017;8: 94692-710.

9. Wu Z, Wang Z, He X, et al. Clinical observation of Zhuyu Annao pill in treating 
40 cases of hemorrhagic apoplexy in acute stage. New J Trad Chin Med 2005;037: 39-41.

10. Rice JE, 3rd, Vannucci RC, Brierley JB. The influence of immaturity on hypoxic-ischemic brain damage in the rat. Ann Neurol 1981;9: 131-41.

11. Nakagomi T, Takagi T, Beppu M, Yoshimura S, Matsuyama T. Neural regeneration by regionally induced stem cells within post-stroke brains: Novel therapy perspectives for stroke patients. World J Stem Cells 2019;11: 452-63.

12. Yang L, Wu D, Wang B, Bu X, Zhu J, Tang J. The effects of hyperbilirubinaemia on synaptic plasticity in the dentate gyrus region of the rat hippocampus in vivo. Arch Med Sci 2020;16: 200-4.

13. Chen W, Hu YQ, Jiang LF, Wu L. Mechanism of action of Zhuyu Annao pill in mice with cerebral intrahemorrhage based on TLR4. Asian Pac J Trop Med 2016;9: 1095-100.

14. Yang S, Wu P, Xiao J, Jiang L. Overexpression of COX6B1 protects against I/Rinduced neuronal injury in rat hippocampal neurons. Mol Med Rep 2019;19: 4852-62.

15. Tan HP, Guo Q, Hua G, Chen JX, Liang JC. Inhibition of endoplasmic reticulum stress alleviates secondary injury after traumatic brain injury. Neural Regen Res 2018;13: 827-36.

16. Choi JB, Kim JH, Lee H, Pak JN, Shim BS, Kim SH. Reactive Oxygen Species and p53 Mediated Activation of p38 and Caspases is Critically Involved in Kaempferol Induced Apoptosis in Colorectal Cancer Cells. J Agric Food Chem 
2018;66: 9960-7.

17. Xu YR, Yang WX. Roles of three Es-Caspases during spermatogenesis and Cadmium-induced apoptosis in Eriocheir sinensis. Aging (Albany NY) 2018;10: 1146-65.

18. Pisani C, Ramella M, Boldorini R, et al. Apoptotic and predictive factors by Bax, Caspases 3/9, Bcl-2, p53 and Ki-67 in prostate cancer after 12 Gy single-dose. Sci Rep 2020;10: 7050.

19. Li L, Liu B. ROCK inhibitor Y27632 protects rats against cerebral ischemia/reperfusioninduced behavioral deficits and hippocampal damage. Mol Med Rep 2019;20: 3395-405.

20. Pinto M, Vempati UD, Diaz F, Peralta S, Moraes CT. Ablation of Cytochrome c in Adult Forebrain Neurons Impairs Oxidative Phosphorylation Without Detectable Apoptosis. Mol Neurobiol 2019;56: 3722-35.

21. Liu Y, Chen J, Jin M, et al. Relationship between changes in mitochondrial function and hippocampal neuronal apoptosis after recurrent convulsion during developmental stage. Exp Ther Med 2018;16: 127-32.

22. Baranov SV, Baranova OV, Yablonska S, et al. Mitochondria modulate programmed neuritic retraction. Proc Natl Acad Sci U S A 2019;116: 650-9.

23. Liang H. Experimental study on secondary brain injury and its protective mechanism after cerebral hemorrhage. Hubei: Huazhong University of Science and Technology; 2013.

24. He Z, Li X, He S. Effects of Naoyi'an Granule on CA1 Regional Nerve Cell 
Withering in Rats with Hemorrhagic Apoplexy. J Hunan Coll Tradit Chin Med 1999;19: 4-6, 14.

25. Li M. Experimental study of hirudin antithrombin in the treatment of cerebral edema after cerebral ischemia in rats. Guangdong: Sun Yat-Sen University; 2004.

26. Jin KY, Xu XJ, Li BL, et al. Study of the Effective Constituents of Danshen Chuanxiongqin Injection Against Hypoxic-Ischemic Brain Injury. Chin J Mod Appl Pharm 2020;37: 1-8.

27. Li JM, Wang YD, Wu MM, Liu T, Gao JL, Li W. Protective effect of chrysophanol on cerebral ischemia reperfusion injury mice by regulating astrocyte-associated protein expression. Chin J Clin Pharmacol 2019;35: 2702-5. 
Table 1 Grouping and dosage of rats

\begin{tabular}{lll}
\hline Group & $\mathrm{n}$ & Dosage \\
\hline MSG & 24 & - \\
MG & 24 & - \\
ZYAN prescription & 24 & $12.50 \mathrm{~g} / \mathrm{kg}$ \\
\hline
\end{tabular}

MSG, mock surgical group; MG, model group; ZYAN, Zhuyu Annao 
Figure legends

Figure 1: Effect of ZYAN prescription on nerve function and spatial LMA in rats with IBI

A) NDS; B) IP results during the detection of spatial LMA through MWM; C) Total swimming distance; D) Number of platform crossings. Notes: ${ }^{\text {a }} P<0.05$ refers to the comparison with MSG; and ${ }^{\mathrm{b}} P<0.05$ refers to the comparison with MG.

LMA, learning and memory ability; NDS, neurodeficit score; MWM, Morris water maze; IP, incubation period; MSG, mock surgical group; MG, model group; ZYAN, Zhuyu Annao

\section{Figure 2: ZYAN prescription reduces the BIS of rats}

A) Gross morphological changes and TTC staining of brain tissues: the white area indicates infarcted area, whereas the red area indicates normal brain tissues; B) comparison of BIS; C) comparison of BWC. A, B, and C refer to MSG, MG, and ZYAN prescription, respectively. Notes: ${ }^{*} P<0.05$ refers to the comparison with MSG, and ${ }^{\#} P<0.05$ refers to the comparison with $\mathrm{MG}$.

TTC, triphenyltetrazolium chloride; BWC, brain water content; MSG, mock surgical group; MG, model group; ZYAN, Zhuyu Annao

Figure 3: Pathomorphology of the hippocampus CA1 area and cortical area in all groups (Nissl staining $\times 400)$

A) Nissl staining of hippocampus CA1 area; B) Nissl staining of cerebral cortex; C) number of positive cells in the hippocampus CA1 area; D) number of positive cells in 
the cerebral cortex. A, B, and C represent MSG, MG, and ZYAN prescription, respectively. Notes: scale: $20 \mu \mathrm{m} ;{ }^{*} P<0.05$ refers to the comparison with $\mathrm{MSG}$; ${ }^{\#} P$ $<0.05$ refers to the comparison with $\mathrm{MG}$.

MSG, mock surgical group; MG, model group; ZYAN, Zhuyu Annao

Figure 4: Detection of cell apoptosis in the hippocampus CA1 area of all groups using the TUNEL method $(\times 400)$

A) Cell apoptosis in the hippocampus CA1 area; B) neuron count in the hippocampus CA1 area; C) AI in the hippocampus CA1 area. A, B, and C represent MSG, MG, and ZYAN prescription. Notes: scale: $20 \mu \mathrm{m}$; ${ }^{\text {a }} P<0.05$ refers to the comparison with MSG; and ${ }^{\mathrm{b}} P<0.05$ refers to the comparison with $\mathrm{MG}$.

MSG, mock surgical group; MG, model group; ZYAN, Zhuyu Annao

Figure 5: Western blotting results showing the expression of apoptosis-related proteins in the hippocampus of all groups

A) Histogram illustrating the cl expressions of apoptosis-related proteins; B) cleaved caspase-3 protein expression; C) Bax protein expression; D) Bcl-2 protein expression; E) p53 protein expression; F) p38 MAPK protein expression. A, B, and C represent MSG, MG, and ZYAN prescription. Notes: ${ }^{a} P<0.05$ refers to the comparison with MSG; and ${ }^{\mathrm{b}} P<0.05$ refers to the comparison with MG.

MSG, mock surgical group; MG, model group 
Figure 6: Detection of cell apoptosis in the hippocampus CA1 and cortical areas in all groups using fluorescent quantitative polymerase chain reaction

A) Cleaved caspase-3 mRNA expression; B) Bax mRNA expression; C) Bcl-2 mRNA expression; D) p53 mRNA expression; E) p38 MAPK mRNA expression. Notes: ${ }^{\text {a }} P$ $<0.05$ refers to the comparison with MSG; and ${ }^{\mathrm{b}} P<0.05$ refers to the comparison with MG.

MSG, mock surgical group; MG, model group 
A
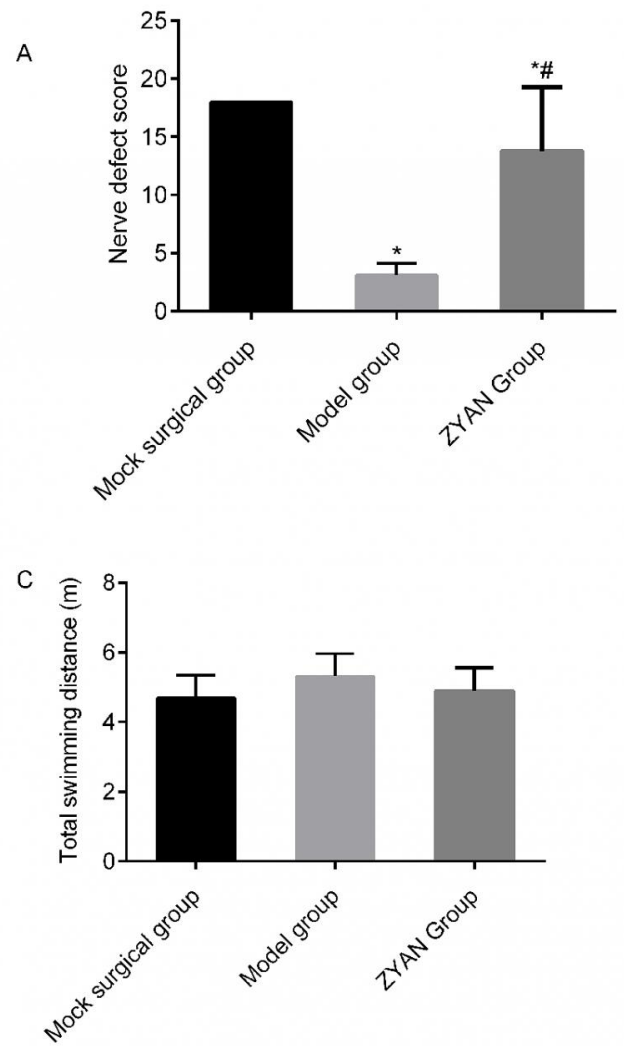

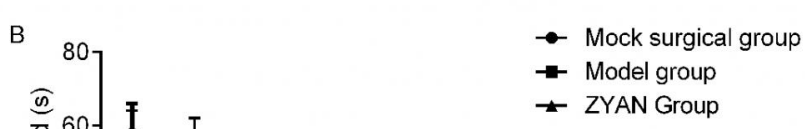

D

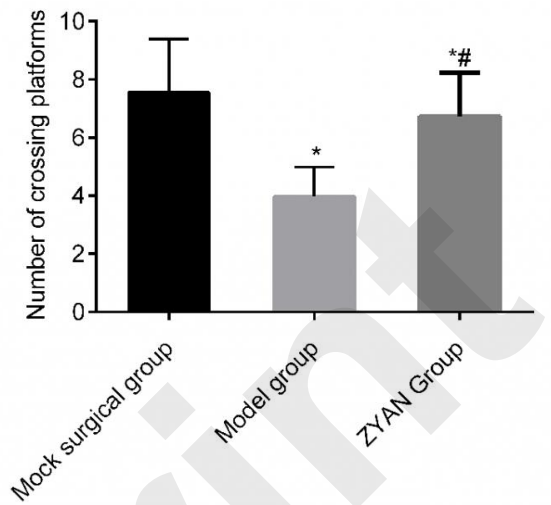


A

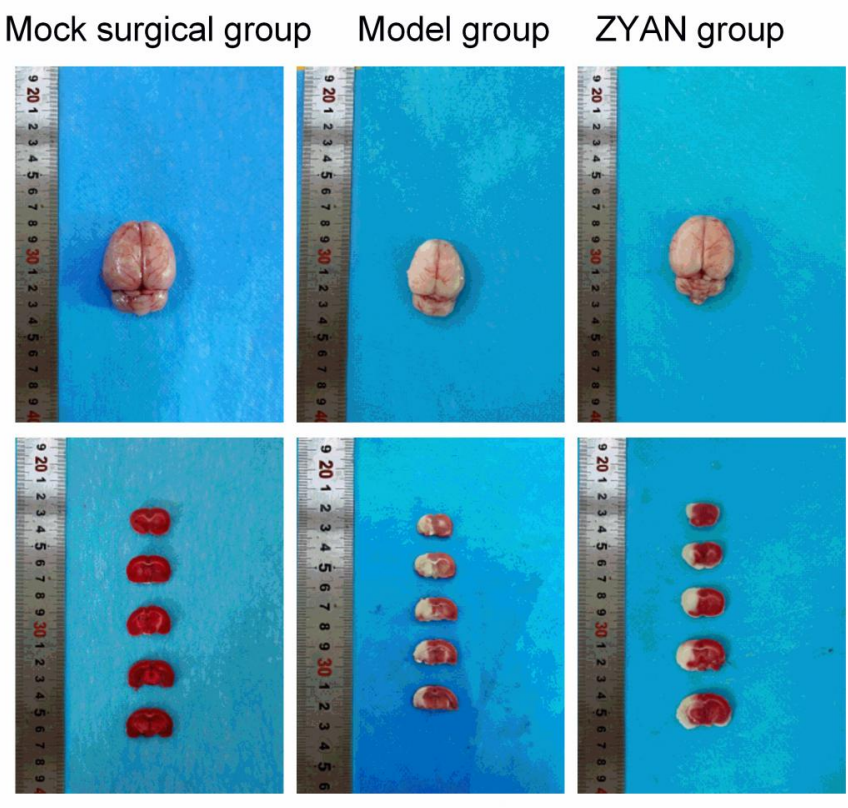

B

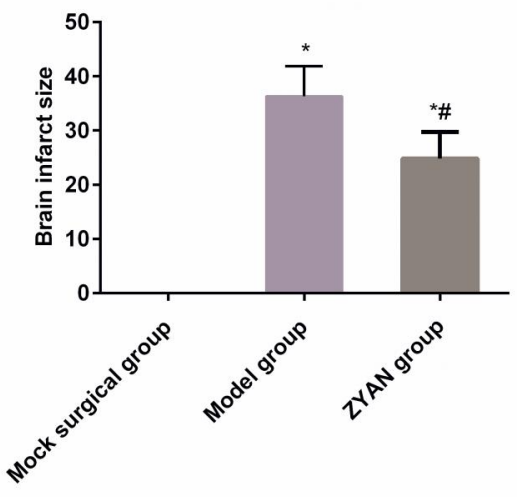

C

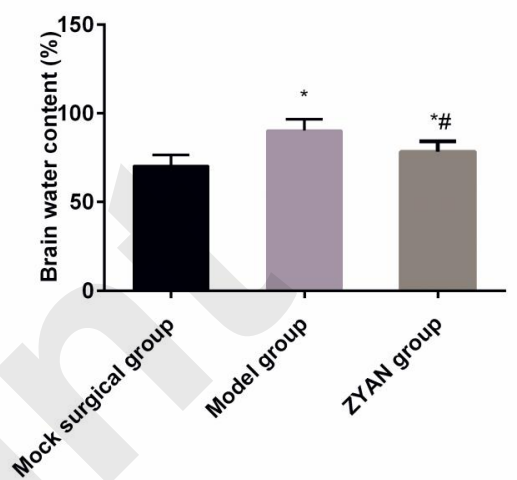



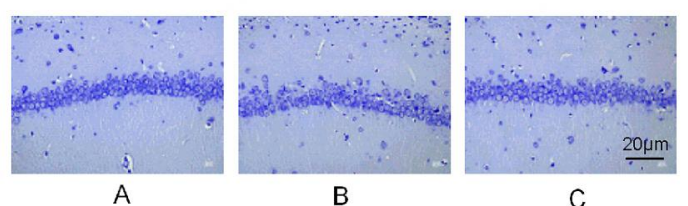

C

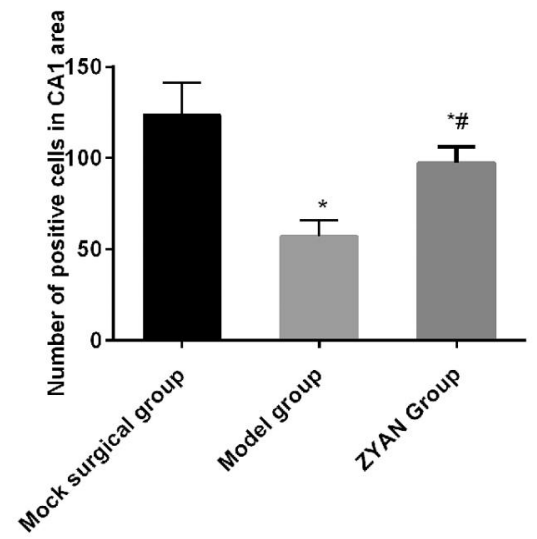

B

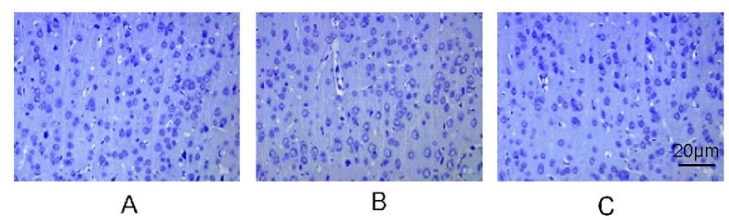

D

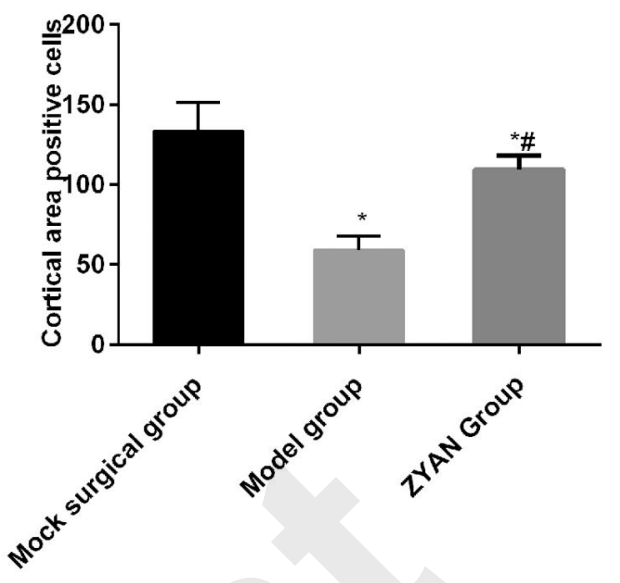


A

Mock surgical group Model group ZYAN group

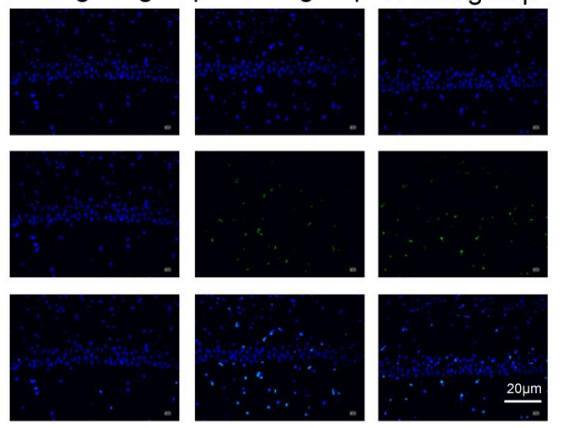

B

C

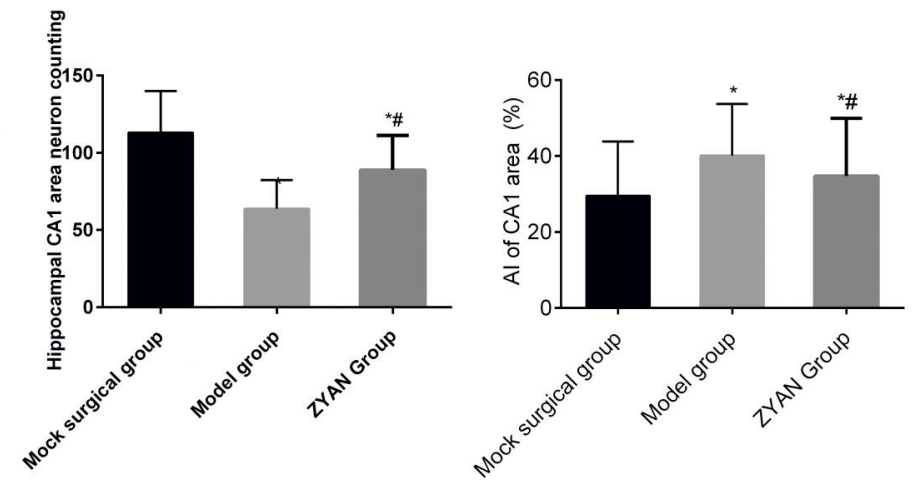


A

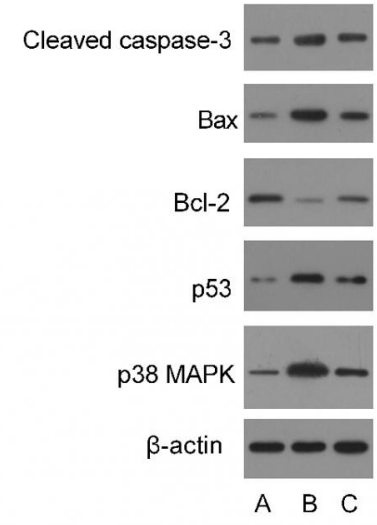

B

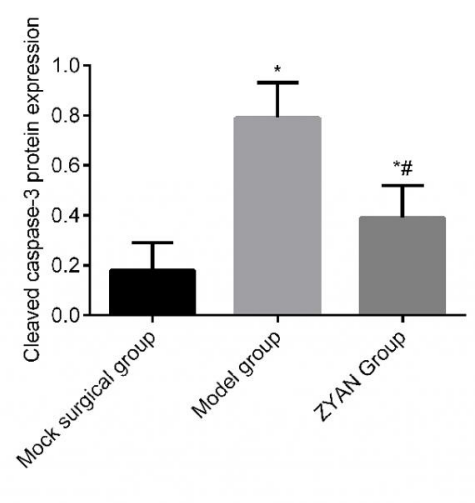

C
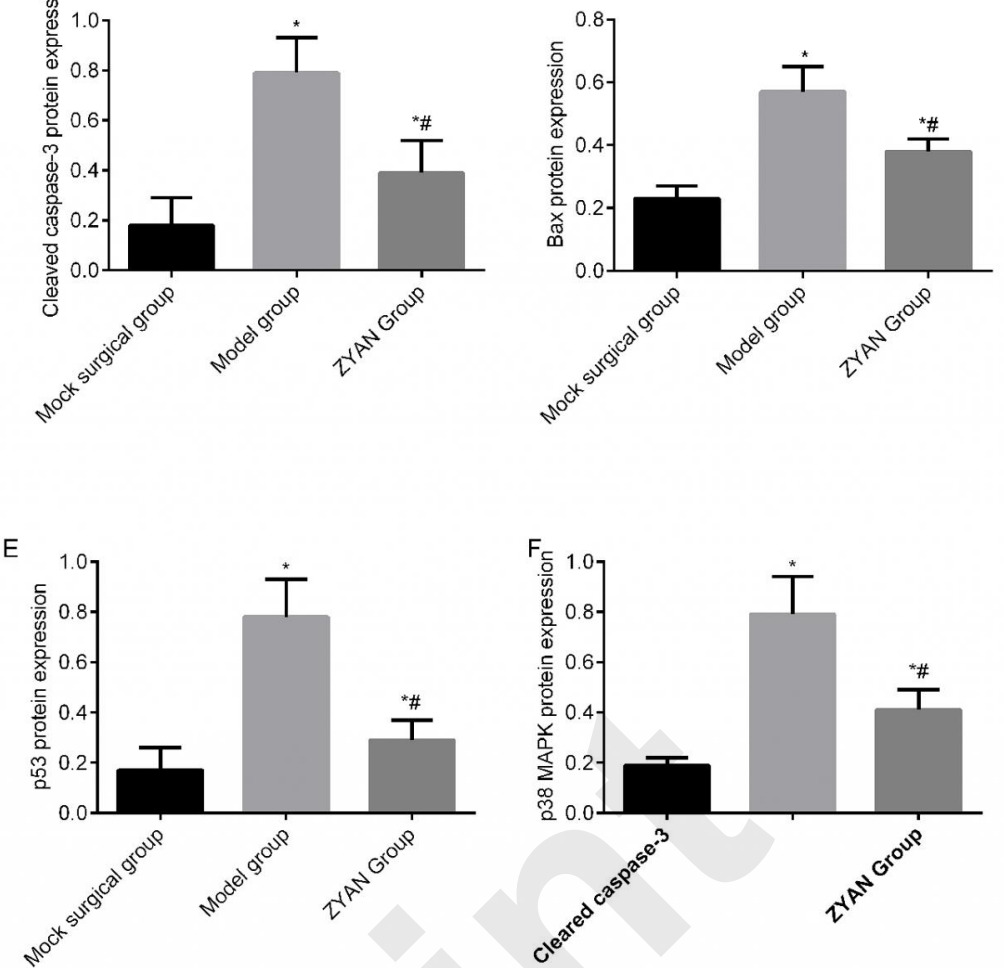

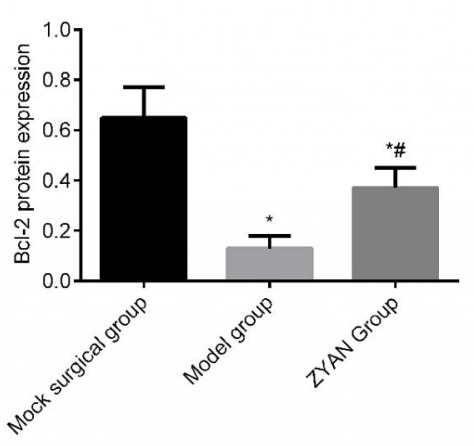


A

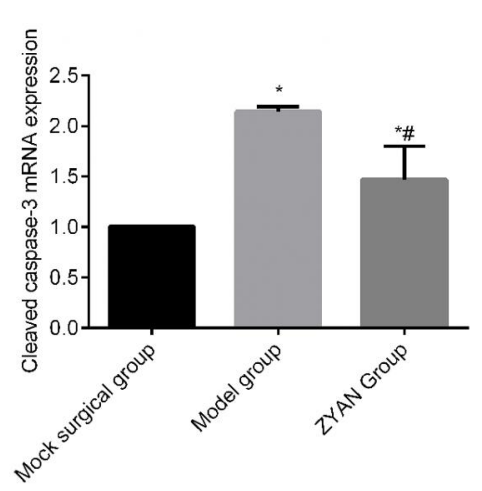

D

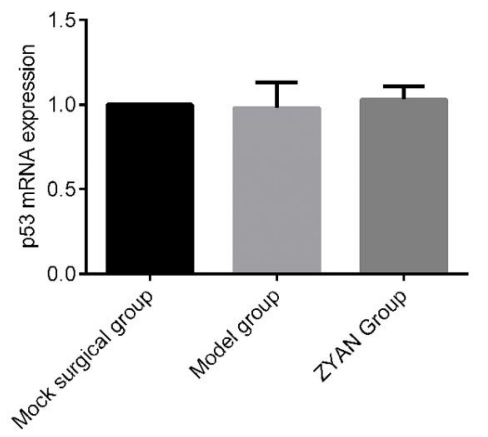

B

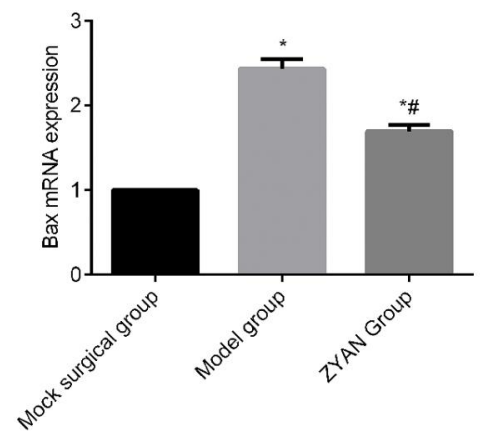

E

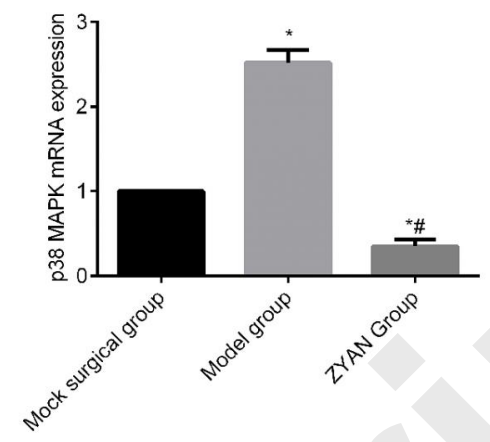

C

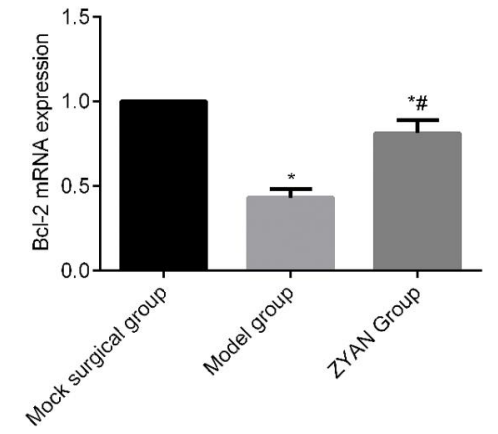

